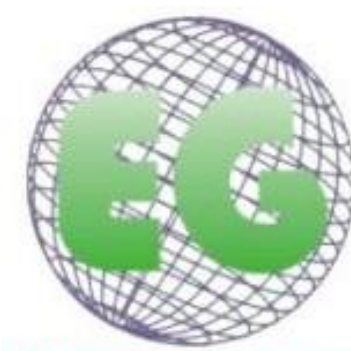

ISSN 1695-6141 $N^{\circ} 56$

\title{
Depresión en pacientes diagnosticados con cáncer en una institución de IV nivel en Montería, Colombia
}

Depression in patients diagnosed with cancer in an IV level institution in Montería, Colombia

\author{
Yolima Judith Llorente Pérez ${ }^{1}$ \\ Beatriz Adriana Macea Tejada ${ }^{2}$ \\ Jorge Luis Herrera Herrera ${ }^{3}$ \\ ${ }^{1}$ Enfermera. Magister en Salud Pública. Docente Universidad del Sinú Elías Bechara Zainúm. Montería. \\ Córdoba. Colombia. yolimallorente@unisinu.edu.co \\ ${ }^{2}$ Enfermera. Docente Universidad del Sinú Elías Bechara Zainúm. Montería. Córdoba. Colombia. \\ ${ }^{3}$ Enfermero. Magister en Enfermería. Docente Universidad del Sinú Elías Bechara Zainúm. Montería. \\ Córdoba. Colombia.
}

\section{http://dx.doi.org/10.6018/eglobal.18.4.354161}

Recibido: $14 / 12 / 2018$

Aceptado: 6/02/2019

\section{RESUMEN:}

Objetivo: Determinar el nivel de depresión en pacientes diagnosticados con cáncer en una institución de IV nivel en Montería, Colombia.

Metodología: Estudio descriptivo, transversal, con enfoque cuantitativo. Se aplicó el instrumento escala de Zung para depresión y una cédula de datos sociodemográficos en pacientes diagnosticados con cáncer, asistentes a una Institución especializada de IV nivel de complejidad de la ciudad de Montería.

Resultados: Los tipos de cáncer más comunes fueron: el de mama 22,2\%; y colon y útero igual representación 18,2\%. En cuanto a la depresión se encontró ligeramente deprimido 49,5\%; moderadamente deprimido 15,7\%; severamente deprimido $1 \%$ y rango normal 33,8\%. Los casos de depresión en sus diferentes categorías según la escala de Zung fueron encontrados en pacientes con cáncer de colon y mama.

Conclusiones: Se encontró que en total $65,2 \%$ de los pacientes presentaban algún grado de depresión. La depresión en pacientes con cáncer es un fenómeno común en las personas con este padecimiento e incidencia es similar en hombres y mujeres, a su vez esta depende de factores tales como el tipo y severidad del cáncer, la edad y el acceso a redes de apoyo tanto para la persona como a la familia.

Palabras claves: Cáncer; depresión; diagnóstico.

\section{ABSTRACT:}

Objective: To determine the level of depression in patients diagnosed with cancer in an IV level institution in Monteria, Colombia.

Methodology: Descriptive, cross-sectional study, with a quantitative approach. The Zung scale instrument for depression and a sociodemographic data card were applied in patients diagnosed with cancer, attending a specialized institution of IV level of complexity in the city of Monteria. 
Results: The most common cancers were: breast cancer 22.2\%; and colon and uterus equal representation $18.2 \%$. Regarding depression, $49.5 \%$ were slightly depressed; moderately depressed $15.7 \%$; severely depressed $1 \%$ and normal range $33.8 \%$. Cases of depression in their different categories according to the Zung scale were found in patients with colon and breast cancer.

Conclusions: It was found that in total $65.2 \%$ of the patients had some degree of depression. Depression in patients with cancer is a common phenomenon in people with this condition and incidence is similar in men and women, in turn this depends on factors such as the type and severity of cancer, age and access to support networks both for the person and the family.

Key words: Cancer; depression; diagnosis

\section{INTRODUCCIÓN}

El cáncer es una patología de interés en salud pública, que se puede presentar sin distinción de sexo, edad, raza, nivel socioeconómico, grado de escolaridad, entre otros. Es una de las principales causas de defunción en el mundo, sobre todo en los países en vía de desarrollo y en el curso o presentación de dicho evento pueden influenciar las determinantes sociales.

Según un informe de la Organización Mundial de la Salud (OMS), es la segunda causa de muerte a nivel mundial; en 2015, ocasionó 8,8 millones de defunciones, casi una de cada seis defunciones en el mundo se debe a esta enfermedad y cerca del $70 \%$ de las muertes por cáncer se registran en países de ingresos medios y bajos. ${ }^{(1)}$

Un tercio de las muertes por cáncer se debe a los cinco principales factores de riesgo conductuales y dietéticos que se pueden intervenir desde atención primaria, específicamente con prevención primaria: índice de masa corporal elevado, ingesta reducida de frutas y verduras, falta de actividad física, consumo de tabaco y consumo de alcohol. El tabaquismo es el principal factor de riesgo y ocasiona aproximadamente el $22 \%$ de las muertes por cáncer. ${ }^{(1)}$ Ante la problemática que genera el cáncer, en 2013, la OMS puso en marcha el Plan de Acción Global para la Prevención y el Control de las Enfermedades No Transmisibles 2013-2020 que tiene como objetivo reducir la mortalidad prematura por el $25 \%$ de cáncer, enfermedades cardiovasculares, diabetes y enfermedades respiratorias crónicas. Algunas de las metas de aplicación voluntaria son especialmente importantes para la prevención del cáncer, como la que propone reducir el consumo de tabaco en un $30 \%$ entre 2014 y 2025. ${ }^{(2)}$

En respuesta a dicho plan, se creó el Plan de Acción para la Prevención y Control de las enfermedades No Transmisibles (ENT) en las Américas 2013 - 2019, que centra sus acciones en las enfermedades de mayor carga ${ }^{(3)}$ para el individuo, familia, comunidad y sistema de salud, partiendo que son eventos de alto impacto en la salud de la colectividad.

Como se ha descrito, es una enfermedad que afecta a cualquier grupo poblacional a nivel mundial, y Colombia no es ajena a dicha situación, dado que se extiende a lo largo de toda la geografía colombiana, en donde vale la pena indicar que algunos habitantes del Departamento de Córdoba, también padecen la enfermedad. Acogidos a ese plan para las Américas, se resalta que Colombia ha reportado un descenso progresivo en las tasas de incidencia y mortalidad por cáncer de cérvix durante los últimos 40 años pasando de 14 muertes por cáncer de cuello uterino por cada 100.000 en 1987 a 7,08 muertes por cada 100000 mujeres al año 2013, cumpliendo así, antes de tiempo la meta establecida y para 2015 y propendiendo por el logro de la 
meta de 5,5 por 100.000 mujeres para el año $2021^{(4-6)}$ y logrado, supervivencia a 5 años de un $45 \%{ }^{(7)}$, compatible con datos internacionales. ${ }^{(8)}$

En el departamento de Córdoba para el periodo 2000-2006, se presentaron 2036 muertes en hombres y en mujeres 2002 casos. En hombres, el $58.8 \%$ del total de defunciones son producidas por cáncer de estómago, pulmón, próstata, colon, recto y leucemias, mientras que para las mujeres el $52.8 \%$ de las muertes se producen por cáncer de cuello de útero, estómago, mama, pulmón, colon ${ }^{(6)}$. Causa del $45 \%$ de las muertes por cáncer en el 2008 , la mayoría consecuencia de cáncer de pulmón, próstata en hombres, y de cáncer de pulmón, mama en mujeres. ${ }^{(9)}$ El 6 de febrero de 2015 en entrevista con el Periódico El Universal, el Secretario de Salud de Córdoba, Edwin Preciado, indicó que no hay estadísticas relacionadas a la muerte por cáncer en esta sección del país, y que además del cáncer de mama o de próstata también se presenta el gástrico, el de piel y el cervicouterino. ${ }^{(10)}$

Por otro lado, se tiene que el hecho de padecer de cáncer puede generar repercusiones a nivel del individuo, familia y comunidad. En el individuo puede afectar la dimensión biopsicosocial toda vez que esta es una enfermedad que altera o deteriora los sistemas corporales, que el hecho de cursarla genera cierta incertidumbre con respecto al tratamiento, pronóstico, cura o muerte, en donde pueden desarrollarse otras patologías derivadas del mismo proceso patológico, como lo puede ser la depresión, la cual se define como un trastorno emocional y mental, que le produce al individuo tristeza y aflicción, que le dificulta su relación con los demás y el medio ambiente que le rodea, es decir que puede alterar su cotidianidad. En la familia la carga que se tiene frente al rol de cuidador informal o cuidador principal y en la sociedad, el impacto que genera por padecer de un evento de interés en salud pública, con alto costo en la atención y tratamiento.

Los individuos que se enfrentan a un cáncer se ven abordados por una serie de respuestas emocionales negativas, en la mayoría de los casos pueden vivir las etapas de duelo: negación, negociación, depresión, ira y finalmente la aceptación del estado de salud. "Enfrentarse a un diagnóstico de cáncer es una de las situaciones más estresantes de la sociedad actual, pocas enfermedades originan tantos problemas psicológicos" (11), debido a que el enfermo debe afrontar el diagnóstico y adaptarse a una serie de cambios en todas sus esferas, y debe tomar decisiones en pro de la mejora de su estado de salud y del mantenimiento de la calidad de vida.

Según lo anterior, el padecer de ciertas enfermedades crónicas como cáncer puede alterar la salud mental de un individuo, toda vez que afecta su rol y genera cambios físicos, sociales, psicológicos, ente otros; y probablemente cambios en los estilos de vida que pueden estar influenciados por las determinantes sociales.

Al hablar de salud mental, se tiene que la OMS, puso en marcha el Plan de Acción Integral sobre Salud Mental 2013 - $2020^{(12)}$, que pretende que se haga valoración integral, potencialización y refuerzo de la salud mental tanto del individuo como de la colectividad, es decir, quiere fortalecer la prevención de trastornos mentales, y que, si una vez se presentan los trastornos, dicho fortalecimiento permita la recuperación del individuo, tanto en su parte social como familiar y personal.

La OMS relaciona que el hecho de padecer trastornos mentales puede agravar 0 influenciar en el desarrollo de ciertos padecimientos como el cáncer, enfermedades cardiacas e infecciosas como VIH/SIDA ${ }^{(12)}$, así mismo, resalta que los factores de riesgo 

transmisibles.

Así mismo, describe que la depresión es un problema de salud pública por la alta carga de enfermedad que genera y relaciona que en el mundo hay 350 millones de personas que se encuentran afectadas por la depresión; siendo el suicidio el peor desenlace. Se estima que cada año se suicidan 800.000 personas en todo el mundo y el grupo de edad más afectado es el de las personas entre 15 y 29 años. ${ }^{(13)}$ Colombia teniendo en cuenta dicho plan, hace seguimiento al comportamiento de los eventos de salud mental y emitió un boletín arrojado por el Sistema Integral de Información de la Protección Social (SISPRO), el cual reporta que las atenciones por depresión se han incrementado desde el año 2009 y el diagnóstico de depresión moderada se realizó en 36.584 atenciones en 2015. ${ }^{(14)}$

De igual forma, en Colombia, la Encuesta Nacional de Salud Mental de 2015 (ENSM) realizada a personas de 12 a 17 años, de 18 a 44 y de 45 años y más mostró el comportamiento de ciertos eventos de salud mental, en donde se relaciona la depresión, la cual se encontró con mayor prevalencia en los adolescentes, representada en un 15,8\%; por regiones, las más afectadas fueron la Central, con una prevalencia de 20,9\% y la Oriental con 16,2\%3; la población de 18 a 44 años tuvo la prevalencia más baja de síntomas de depresión y las mujeres las más afectadas ${ }^{(15)}$ y el Departamento de Córdoba tuvo 113 casos de persona diagnosticadas con depresión modera. ${ }^{(14)}$ Por otra parte, Colombia para fortalecer la Salud Mental de los individuos, incluyó como dimensión prioritaria la convivencia social y salud mental en el Plan Decenal de Salud Pública 2012 - 2021, en donde se persigue atención integral para toda la población y mejoras en la calidad de vida, en donde el individuo pueda hacer disfrute de su cotidianidad, dimensión que también es respaldada a través de la Ley 1616 de 2013, que busca garantizar la promoción de la salud mental dentro del territorio nacional.

Teniendo en cuenta la situación del País y la del Departamento de Córdoba para cáncer y depresión como enfermedades no transmisibles surge la siguiente pregunta de investigación ¿Cuál es el nivel de depresión en pacientes diagnosticados con cáncer en una institución de IV nivel en Montería, Colombia?

El hecho de identificar el nivel de depresión en pacientes con cáncer puede ayudar a la toma de decisiones en salud pública a los diferentes entes de control como lo es la Secretaria de Salud Departamental, en el sentido que se pueden promover programas en pro de mejorar la calidad de vida de los pacientes, que busquen generar mayor seguridad y confianza en el diagnóstico y tratamiento oportuno, y disminuir de esta forma desenlaces fatales; así mismo, les permitirá evaluar y mejorar las políticas de salud pública en materia de enfermedades no transmisibles, toda vez que ambos eventos generan alta discapacidad y llevan a la muerte.

El estudio fue realizado en una IPS de IV nivel de atención en la Ciudad Montería Córdoba, en donde se utilizaron las estadísticas actualizadas y la escala ZUNG, la cual indica presencia y severidad de síntomas depresivos, somáticos y cognitivos, como también la intensidad y frecuencia de los mismos. Dicha escala permitió identificar la existencia e intensidad del estado de depresión en el paciente, lo que resalta la necesidad de intervenir estas personas con un equipo interdisciplinario, con el objetivo de mejorar o mantener su calidad de vida a lo largo de todo el proceso de la enfermedad. La investigación también suministra herramientas que pueden ser utilizadas tanto a nivel 
social como político, en donde los mayores beneficiarios de la puesta en marcha de nuevas políticas serán el individuo, familia y comunidad.

\section{OBJETIVOS}

\section{General}

Determinar el nivel de depresión en pacientes diagnosticados con cáncer en una institución de IV nivel en Montería, Colombia.

\section{Específicos}

- Caracterizar la población según variables de interés: sexo, edad, procedencia, estado civil y grado de escolaridad.

- Identificar los tipos de cáncer en la población objeto de estudio

- Identificar el nivel de depresión en la población objeto de estudio

- Relacionar el nivel de depresión con las variables de sexo, edad y tipos de cáncer.

\section{MATERIAL Y MÉTODO}

\section{Tipo de estudio}

Esta investigación se clasifica como de alcance descriptivo, transversal, cuyo propósito fue describir el nivel de ansiedad de pacientes diagnosticados con cualquier tipo de cáncer, asistente a una Institución especializada de IV nivel de complejidad de la ciudad de Montería; teniendo en cuenta el nivel de medición utilizado corresponde a un diseño con enfoque cuantitativo.

\section{Unidad de análisis}

La unidad de análisis la conformo el nivel de ansiedad, encontrado en los pacientes participantes en el estudio, así como las variables sociodemográficas seleccionadas para caracterizar a la muestra objeto de estudio.

\section{Muestra y muestreo}

La muestra la conformaron 198 sujetos, esta se calculó partiendo de una población general asistente a la institución y accesible a los investigadores de 200 pacientes, con un nivel deseado de confianza de $99 \%$ y error máximo aceptable de $1 \%$

El muestreo fue de tipo no probabilístico o intencional, se incluyeron en el estudio personas en el rango de edad de 18 a 50 años de edad, sin ningún tipo de limitación cognoscitiva o verbal que le impidiera responder las preguntas que contemplaba el instrumento seleccionado, así mismo que manifestaran su deseo explícito de participar en la investigación.

El instrumento seleccionado para conocer el nivel de ansiedad fue la escala de Zung para depresión; esta ha sido validada en el contexto colombiano por Campo et al, (16) quien encontró un Alpha de Cronbach de 0,85, sensibilidad de 94,7\% y correlación 
test retest de 0,848 ( $p<0,01$ ), lo que la convierte en un instrumento con propiedades psicométricas confiables.

\section{RESULTADOS}

Los participantes en el estudio fueron en igual porcentaje hombres y mujeres en edades comprendidas en el rango de 41 a 50 años, procedentes del área urbana de la ciudad de Montería, primando en ellos el estado civil de unión libre y un grado de escolaridad de bachillerato completo.

A continuación, se presenta los datos obtenidos teniendo en cuenta el tipo de cáncer diagnosticado en la muestra seleccionada.

\section{Tabla 1. Distribución por tipos de cáncer}

\begin{tabular}{lcc} 
Tipo de Cáncer & Frecuencia & Porcentaje \\
\hline Colon & 36 & 18,2 \\
\hline Leucemia & 12 & 6,1 \\
\hline Mama & 44 & 22,2 \\
\hline Estomago & 6 & 3,0 \\
\hline Útero & 36 & 18,2 \\
\hline Próstata & 19 & 9,6 \\
\hline Cerebro & 5 & 2,5 \\
\hline Mieloma múltiple & 5 & 2,5 \\
Columna & 4 & 2,0 \\
\hline Linfoma no Hogking & 4 & 2,0 \\
\hline Pulmón & 12 & 6,1 \\
\hline Laringe & 9 & 4,5 \\
Riñón & 2 & 1,0 \\
\hline Condrosarcoma & 1 & 0,5 \\
\hline Piel & 2 & 1,0 \\
\hline Hígado & 1 & 0,5 \\
\hline TOTAL & 198 & 100,0 \\
\hline
\end{tabular}

Fuente: cedula de datos demográficos y clínicos

Se observa que el cáncer más común fue el cáncer de mama con 22,2\%, seguido del cáncer de colon y útero, los cuales comparten igual porcentaje 18,2\%, en menor proporción el cáncer de hígado y el Condrosarcoma con $0,5 \%$ respectivamente. 
Tabla 2. Nivel de depresión

Nivel de Depresión

$\mathbf{N}^{\circ}$

$\%$

\begin{tabular}{lcc}
\hline Rango normal & 67 & 33,8 \\
\hline Ligeramente deprimido & 98 & 49,5 \\
\hline Moderadamente deprimido & 31 & 15,7 \\
\hline Severamente deprimido & 2 & 1,0 \\
\hline TOTAL & 198 & 100 \\
\hline
\end{tabular}

Fuente: escala de Zung

Como dato relevante se obtuvo que $49,5 \%$ de la muestra se encontrara ligeramente deprimido; $15,7 \%$ moderadamente deprimido y $1 \%$ severamente deprimido, lo que quiere decir que en total $65,2 \%$ de los pacientes presentaban algún grado de depresión. En contraposición a ello el $33,8 \%$ se encontraba en rango normal o sin depresión.

Tabla 3. Nivel de depresión por sexo

NIVEL DE DEPRESIÓN Y SEXO

\begin{tabular}{lcccc}
\hline Nivel de Depresión & Femenino & $\%$ & Masculino & $\%$ \\
\hline Rango normal & 25 & 25,3 & 42 & 42,4 \\
\hline Ligeramente deprimido & 66 & 66,7 & 32 & 32,3 \\
\hline Moderadamente deprimido & 6 & 6,1 & 25 & 25,3 \\
\hline Severamente deprimido & 2 & 2,0 & 0 & 0,0 \\
\hline TOTAL & 99 & 100 & 99 & 100 \\
\hline
\end{tabular}

Fuente: escala de Zung

La mayoría de casos de depresión se presentaron en el sexo femenino, en donde se encontró ligeramente deprimido $66,7 \%$, moderadamente deprimido $6,1 \%$, de igual forma fue el único grupo en el que se encontraron casos de severos de depresión, en su totalidad el $74,8 \%$ de la muestra femenina presento depresión; en contraposición el sexo masculino presento en ligeramente deprimido $32,3 \%$, y en moderadamente deprimido $25,3 \%$, es decir que solo $57,6 \%$ presentaron depresión. 
Tabla 4. Depresión por grupo de edad

\begin{tabular}{|c|c|c|c|c|c|c|}
\hline \multicolumn{7}{|c|}{ NIVEL DE DEPRESIÓN Y EDAD } \\
\hline \multirow[t]{2}{*}{ Nivel de depresión } & 20-30 años & \multirow[t]{2}{*}{$\%$} & \multirow[t]{2}{*}{$31-40$ años $(n=55)$} & \multirow[t]{2}{*}{$\%$} & \multirow{2}{*}{$\begin{array}{c}\text { 41-50 años } \\
(n=135)\end{array}$} & \multirow[t]{2}{*}{$\%$} \\
\hline & $(n=8)$ & & & & & \\
\hline Rango normal & 3 & 37,5 & 22 & 40 & 42 & 31,1 \\
\hline Ligeramente deprimido & 3 & 37,5 & 27 & 49,1 & 68 & 50,4 \\
\hline Moderadamente deprimido & 2 & 25 & 6 & 10,9 & 23 & 17,0 \\
\hline Severamente deprimido & 0 & 0 & 0 & 0,0 & 2 & 1,5 \\
\hline TOTAL & 8 & 100 & 55 & 100 & 135 & 100 \\
\hline
\end{tabular}

Fuente: escala de Zung y cedula de datos

Se encontró que el rango de edad que presento mayor depresión, fue el rango de 41 a 50 años con un porcentaje total de $68,9 \%$, siendo el nivel de depresión más encontrado ligeramente deprimido con $50,4 \%$ y fue en este rango que se ubicaron los dos casos de depresión severa. El grupo de edad de 20 a 30 años fue el que presento menos casos de depresión.

Tabla 5. Nivel de depresión por tipo de cáncer

\begin{tabular}{|l|c|c|c|c|c|c|c|c|c|}
\hline \multicolumn{2}{|c|}{ NIVEL DE DEPRESIÓN VS TIPO DE CÁNCER } \\
\hline $\begin{array}{l}\text { TIPO DE } \\
\text { CÁNCER }\end{array}$ & $\begin{array}{c}\text { Rango } \\
\text { normal }\end{array}$ & $\%$ & $\begin{array}{c}\text { Ligerament } \\
\text { e } \\
\text { deprimido }\end{array}$ & $\%$ & $\begin{array}{c}\text { Moderada } \\
\text { mente } \\
\text { deprimido }\end{array}$ & $\%$ & $\begin{array}{c}\text { Severament } \\
\text { e deprimido }\end{array}$ & $\%$ & Total \\
\hline Colon & 9 & 13,4 & 18 & 18,4 & 9 & 29,0 & 0 & 0,0 & 36 \\
\hline Leucemia & 8 & 11,9 & 2 & 2,0 & 2 & 6,5 & 0 & 0,0 & 12 \\
\hline Mama & 15 & 22,4 & 16 & 16,3 & 12 & 38,7 & 1 & 50,0 & 44 \\
\hline Estomago & 1 & 1,5 & 4 & 4,1 & 1 & 3,2 & 0 & 0,0 & 6 \\
\hline Útero & 8 & 11,9 & 28 & 28,6 & 0 & 0,0 & 0 & 0,0 & 36 \\
\hline Próstata & 10 & 14,9 & 9 & 9,2 & 0 & 0,0 & 0 & 0,0 & 19 \\
\hline Cerebro & 2 & 3,0 & 2 & 2,0 & 1 & 3,2 & 0 & 0,0 & 5 \\
\hline $\begin{array}{l}\text { Mieloma } \\
\text { múltiple }\end{array}$ & 3 & 4,5 & 2 & 2,0 & 0 & 0,0 & 0 & 0,0 & 5 \\
\hline Columna & 2 & 3,0 & 2 & 2,0 & 0 & 0,0 & 0 & 0,0 & 4 \\
\hline $\begin{array}{l}\text { Linfoma } \\
\text { Hogking }\end{array}$ & 2 & 3,0 & 0 & 0,0 & 2 & 6,5 & 0 & 0,0 & 4 \\
\hline Pulmón & 3 & 4,5 & 6 & 6,1 & 3 & 9,7 & 0 & 0,0 & 12 \\
\hline Laringe & 3 & 4,5 & 5 & 5,1 & 1 & 3,2 & 0 & 0,0 & 9 \\
\hline Riñón & 0 & 0,0 & 2 & 2,0 & 0 & 0,0 & 0 & 0,0 & 2 \\
\hline $\begin{array}{l}\text { Condrosarco } \\
\text { ma }\end{array}$ & 0 & 0,0 & 1 & 1,0 & 0 & 0,0 & 0 & 0,0 & 1 \\
\hline Piel & 1 & 1,5 & 0 & 0,0 & 0 & 0,0 & 1 & 50,0 & 2 \\
\hline Hígado & 0 & 0,0 & 1 & 1,0 & 0 & 0,0 & 0 & 0,0 & 1 \\
\hline$N^{\circ}$ & 67 & 100 & 98 & 100 & 31 & 100 & 2 & 100 & 198 \\
\hline
\end{tabular}

Fuente: escala de Zung y cedula de datos 
Como dato relevante se obtuvo que los casos de depresión en sus diferentes categorías según la escala de Zung, fueron encontrados en pacientes diagnosticados con cáncer de colon y mama; en cuanto a la categoría de depresión más frecuente, en colon fue levemente deprimido con $18,4 \%$, y en cáncer de mama moderadamente deprimido $38,7 \%$.

\section{DISCUSIÓN}

En el informe sobre situación del cáncer en Colombia para el año $2015,{ }^{(17)}$ se estimaron cerca de 14 millones de casos nuevos de cáncer diagnosticados en el mundo para 2012; de estos 71.442 casos ocurrieron en Colombia, así mismo dichas proyecciones dejan entrever un empeoramiento de esta problemática ya que en el 2015 se esperan 79.660 personas con cáncer en el país, con una incidencia mayor en mujeres menores de 65 años, igualando los datos en cuanto a edad obtenidos en esta investigación y en 2035 cerca del doble de casos (152.901) a expensas de los hombres mayores de 65 años, la característica de edad en este estimativo difiere de lo encontrado por los investigadores ya que el límite máximo de edad hallado fue de 50 años, vale la pena destacar que no se discriminó la edad por sexo en la muestra estudiada.

En este sentido y contrastando lo descrito con los resultados encontrados en la presente investigación se evidencia diferencia en cuanto al sexo ya que el comportamiento de esta enfermedad en Colombia ha sido mayor en los últimos años y ha afectado en proporciones mayores a mujeres.

En cuanto al nivel educativo se refiere, autores han encontrado que las tasas totales de mortalidad por cáncer disminuyen más rápidamente entre las personas con educación superior, factor proyector encontrado en esta investigación ya que en la muestra estudiada primó en cuanto a grado de escolaridad el bachillerato completo, sin embargo, fue menor la proporción de personas con formación en educación superior.

Alarcón et al, ${ }^{(18)}$ mediante un estudio descriptivo - transversal que incluyo a 11.500 pacientes con cáncer de 16 ciudades de Colombia; durante los años 2010 a 2013, encontraron que los tipos de cáncer más prevalentes fueron en su orden: mama $(116,2)$, otros próstata $(79,4)$, piel $(61,3)$, tiroides $(56,8)$, cérvix $(50,3)$, colon-recto $(39,7)$, los datos obtenidos durante esta investigación coinciden con los expuestos por este autor, ya que los tipos de cáncer más frecuentes fueron en orden según el número de casos mama, colon, útero y próstata, ocupando el primero lugar el de mama así como en la investigación citada.

Al hacer referencia al comportamiento de la depresión en los pacientes diagnosticados con cáncer, se tiene que es un fenómeno ampliamente estudiado ${ }^{(19)}$ y los resultados disponibles en la literatura consultada difieren de un autor a otro, así mismo depende en gran medida del tipo de cáncer, la ubicación geográfica, el acceso a tratamiento y el apoyo con el que pueda contar la persona que lo padece.

En este sentido, al comparar los resultados obtenidos con investigaciones previas, se encuentra que la depresión es el trastorno más común en pacientes con prevalencia de 22 a $29 \%$, (20) situación claramente concordante con los datos que se obtuvieron al 
estudiar la muestra objeto, donde $65,2 \%$ de los pacientes presentaron grados de depresión significativa.

De igual forma Valencia, ${ }^{(21)}$ coincide con estas cifras asociadas a la depresión por cáncer que permiten vislumbrar una tendencia al aumento, además señala que dicho trastorno es más común en personas con cáncer agresivo y discapacitante, en la presente investigación, cuando se analiza la depresión según el tipo de cáncer, se evidencia que esta fue más común en cáncer de colon y mama, los cuales son reconocidos no solo como agresivos y de alto impacto, sino también discapacitantes y que causan deterioro en la imagen corporal, perpetuando los síntomas de depresión.

\section{CONCLUSIONES}

En el presente estudio la muestra estudiada se caracterizó por estar compuesta por hombres y mujeres en igual porcentaje, con un rango de edades entre los 41 a 50 años, que procedían del área urbana de la ciudad y que no sobrepasan el grado de bachillerato completo.

Se encontró que en total $65,2 \%$ de los pacientes presentaban algún grado de depresión; la mayoría de casos de depresión se presentaron en el sexo femenino, en donde se encontró ligeramente deprimido 66,7\%, moderadamente deprimido 6,1\%, de igual forma fue el único grupo en el que se encontraron casos severos de depresión.

Se puede decir que la depresión en pacientes con cáncer es un fenómeno común en las personas con este padecimiento e incidencia es similar en hombres y mujeres, a su vez esta depende de factores tales como el tipo y severidad del cáncer, la edad y el acceso a redes de apoyo tanto para la persona como a la familia.

\section{REFERENCIAS}

1. Ferlay J, Soerjomataram I, Ervik M, Dikshit R, Eser S, Mathers $C$ et al. Globocan 2012 v1.0, Cancer incidence and mortality worldwide: larc Cancerbase no. 11. Lyon, France: Centro Internacional de Investigaciones sobre el Cáncer; 2013.

2. Stewart B., Wird C. Informe mundial sobre el cáncer, 2014, Agencia Internacional para la investigación sobre el cáncer, OMS, ISBN 978-92-8320429-9, disponible en: http://www.iarc.fr/en/publications/books/wcr/wcrorder.php, consultado el día 10 agosto de 2018.

3. Organización Mundial de la Salud. Informe sobre la situación mundial de las enfermedades no transmisibles 2010 [internet]. Ginebra: OMS; 2011 [resumen de orientación en español, informe completo en inglés, consultado el 1 de octubre del 2018]. se encuentra en: http://www.who.int/nmh/publications/ncd_report2010/es/index.html

4. Ministerio de salud y Protección Social - Instituto Nacional de cancerología. Plan Decenal de Cáncer 2012-2021, Bogotá. 2012

5. Consejo Nacional de política económica y social. (2011). CONPES 140, Plan Nacional de desarrollo. Bogotá

6. Piñeros M, pardo C, Gamboa O, Hernández G. Atlas de mortalidad por cáncer en Colombia. Instituto Nacional de Cancerología, instituto Geográfico Agustin Codazzi. Bogotá: Imprenta nacional; 2010. 
7. Bravo LE, carrascal E, García LE, Collazos T, Gutierrez A. Supervivencia de cáncer de cuello uterino en Cali: un estudio de base poblacional. Informe técnico INC - univalle Edición Cali. 2006.

8. Quinn MA, Benedett JL, Odicino F, Maisonnueve P, Beller U, Creasman. Carcinoma of the cérvix uterin. FIGO 6th Annual report on the results of treatment in gynecological cancer. Int J Gynecol Obstetric 2006;95 (suppl. 1) S43-103

9. Sanabria Russi, María; Mendoza Gonzalez, Natalia. Perfil epidemiológico de pacientes con cáncer en tratamiento ambulatorio en una ips de bogotá, Universidad CES, pag:1-64

10. Serrano N. Aumenta índice de muertes por cáncer de mama en Córdoba, 2015, Periódico El Universal, disponible en: http://www.eluniversal.com.co/regional/cordoba/aumenta-indice-de-muertespor-cancer-de-mama-en-cordoba-184066 , consultado el día 20 de agosto de 2018.

11. Alonso Fernández, María Del Carmen. Bastos Flores, Amparo. Intervención Psicológica en personas con cáncer. Artículo. Consultado el día 28 de julio 2018. Disponible en milacahue.com/. en el documento anterior (18-05-2015).

12. Plan de acción integral sobre salud mental 2013 - 2020, disponible en http://apps.who.int/iris/bitstream/handle/10665/97488/9789243506029_spa.pdf? sequence $=1$ consultado 10 noviembre de 2018

13. Organización Mundial de la Salud, OMS, disponible en: http://www.who.int/topics/depression/es/ [Consultado el 14 de noviembre de 2018].

14. Boletín de salud mental Depresión Subdirección de Enfermedades No Transmisibles

15. Ministerio de Salud y Protección Social y Colciencias. Encuesta Nacional de Salud Mental 2015, tomo I. Bogotá 2015.

16. Campo-Arias, A, Díaz-Martínez, LA, Rueda-Jaimes, GE, Barros-Bermúdez, JA. Validación de la escala de Zung para depresión en universitarias de Bucaramanga, Colombia. Revista Colombiana de Psiquiatría [Internet]. 2005;XXXIV(1):54-62. Recuperado de: https://www.redalyc.org/articulo.oa?id=80628403005

17. Instituto Nacional de Cancerologia - ESE, Colombia. Análisis de Situación del Cáncer en Colombia 2015 [Internet [citado el 2 de diciembre de 2018]. Disponible

http://www.cancer.gov.co/Situacion_del_Cancer_en_Colombia_2015.pdf

18. Alarcón Blanca, Guarín Natalia, Muñoz-Galindo Iván, Díaz Jorge, Arévalo Helbert. Prevalencia del cáncer en una aseguradora en salud en Colombia, 2013. rev.colomb.cancerol. [Internet]. 2015 Oct [cited 2018 Dec 13]; 19(4): 210-221. Available from: http://www.scielo.org.co/scielo.php?script=sci_arttext\&pid=S012390152015000400004\&lng=en. http://dx.doi.org/10.10167j.rccan.2015.07.001.

19. Rodríguez, V. P., Amboage, A. M., Blázquez, M. H., Torres, M. Á. G., \& Gaviria, M. (2015). Depresión y cáncer: una revisión orientada a la práctica clínica. Revista Colombiana de Cancerología, 19(3), 166-172.

20. De Paula, J., Sonobe, H., Fontao, M., \& Sawada, N. (2012). Síntomas de depresión en los pacien-tes con cáncer de cabeza y cuello en tratamiento radioterápico: un estudio prospectivo. Revis-ta Latinoamericana de Enfermagem, 20(2).

21. Valencia Lara, Sandra Carolina. Depresión en personas diagnosticadas con cáncer. Diversitas, [S.I.], v. 2, n. 2, p. 241-257, jun. 2006. ISSN 2256-3067. 
Disponible en: $<$ http://revistas.usantotomas.edu.co/index.php/diversitas/article/view/132/186>. Fecha de acceso: 14 dic. 2018 doi:https://doi.org/10.15332/s17949998.2006.0002.05. 